\title{
Robust Time-Frequency Distributions with Complex-Lag Argument
}

\author{
Nikola Žarić, Irena Orović, and Srdjan Stanković \\ Faculty of Electrical Engineering, University of Montenegro, Podgorica 20000, Montenegro \\ Correspondence should be addressed to Nikola Žarić, zaric@ac.me \\ Received 30 December 2009; Accepted 1 March 2010 \\ Academic Editor: Igor Djurović \\ Copyright (C) 2010 Nikola Žarić et al. This is an open access article distributed under the Creative Commons Attribution License, \\ which permits unrestricted use, distribution, and reproduction in any medium, provided the original work is properly cited. \\ The robust time-frequency distributions with complex-lag argument are proposed. They can provide an accurate estimation of \\ fast varying instantaneous frequency in the presence of noise with heavy-tailed probability density function. The L-estimate form \\ of this distribution is defined and it includes the L-estimate form of Wigner distribution as a special case. A modification for \\ multicomponent signal representation is proposed, as well. Theoretical considerations are illustrated by the examples.
}

\section{Introduction}

Nonstationary signals such as speech, radar, seismic, sonar, and biomedical signals can be found in many practical applications. Due to time-varying spectra of these signals, time-frequency analysis has been used in their analysis. For different types of signals, various time-frequency distributions (TFDs) have been proposed [1-5].

In real applications we deal with signals corrupted by noise. If noise is additive with Gaussian probability density function (pdf), the standard time-frequency distributions represent a maximum likelihood (ML) estimate [6]. However, if the signal is corrupted by noise with heavy-tailed pdf (usually caused by environmental or human-made activities), the standard TFDs produce poor results. Consequently, the robust time-frequency distributions have been introduced [6-12]. The simplest and the most commonly used robust time-frequency representation is the robust shorttime Fourier transform (STFT). The marginal median robust STFT has been introduced as an ML estimate of signals with Laplacian noise [8]. This form can also be successfully used for other types of heavy-tailed noises. The L-estimate robust STFT is introduced for signals with a mixture of Gaussian and impulse noises [9]. As in the case of the standard STFT, the main drawback of the robust STFT is a poor time-frequency resolution. In order to improve the timefrequency concentration, the robust forms of the Wigner distribution (WD) have been introduced [9-12]. They can provide an ideal concentration for signals with a linear instantaneous frequency (IF). However, for multicomponent signals the cross-terms appear. The robust S-method that combines good properties of the STFT and the WD has been introduced to provide a cross-terms free representation [12]. However, it cannot provide good concentration for signals with fast varying IF. Thus, the time-frequency distributions with complex-lag argument have been used to estimate nonlinear and fast IF variations [13-20]. Similarly as other TFDs, these distributions provide poor signal representation in the presence of heavy-tailed noise.

In this paper we propose a robust form of the Nthorder complex-lag time-frequency distribution (CTD). An arbitrary high concentration can be achieved by increasing the distribution order $N$. The standard CTD has been defined as convolution of the WD and the Fourier transform of the higher order complex-lag moment, called concentration function (CF) $[17,18]$. Similarly, the robust CTD can be obtained as convolution of the robust WD and CF forms. Additionally, a cross-terms free robust complex-lag timefrequency distribution is proposed for multicomponent signals.

The paper is organized as follows. The elementary theory behind linear and quadratic robust time-frequency representations is presented in Section 2. The Nth order robust time-frequency distributions with complex-lag argument are 
proposed in Section 3. The advantages of the proposed distributions are proven through various examples in Section 4. Concluding remarks are given in Section 5.

\section{Theoretical Background}

2.1. Robust Short-Time Fourier Transform. Consider the noisy signal $x(n)=s(n)+v(n)$, where $s(n)$ is a complexvalued signal corrupted with complex-valued noise $v(n)$. The STFT can be obtained as a solution of the following optimization problem:

$$
\operatorname{STFT}(n, k)=\arg \min _{\mu} \sum_{m=-N_{s} / 2}^{N_{s} / 2-1} F(e(n, k, m))
$$

where $F(e)$ is the loss function, while $e(n, k, m)$ is the error function:

$$
e(n, k, m)=x(n+m) e^{-j 2 \pi m k / N_{s}}-\mu .
$$

The number of samples within the window is denoted as $N_{s}$, while $\mu$ represents complex-valued optimization parameter. In the case of the loss function $F(e)=|e|^{2}$, the standard STFT is obtained:

$$
\begin{aligned}
& \operatorname{STFT}_{S}(n, k) \\
& =\frac{1}{N_{s}} \sum_{m=-N_{s} / 2}^{N_{s} / 2-1} x(n+m) e^{-j 2 \pi m k / N_{s}} \\
& =\operatorname{mean}\left\{x(n+m) e^{-j 2 \pi m k / N_{s}}, m \in\left[-\frac{N_{s}}{2}, \frac{N_{s}}{2}\right)\right\} .
\end{aligned}
$$

The quadratic loss function is the ML estimate of a signal corrupted with Gaussian noise. Hence, the standard STFT is applicable in this case. Nevertheless, when impulse noise is present, the quadratic loss function yields poor results. Therefore, other loss functions should be used. For instance, it has been shown that the loss function $F(e)=|e|$ exhibits a robust behavior for heavy-tailed noise (e.g., Cauchy noise, Laplacian noise) $[6,11,21]$. The implicit solution of the optimization problem in (1) for $F(e)=|e|$ requires a computationally demanding iterative procedure. In order to avoid such an iterative procedure, the marginal median estimate, with the loss function $F(e)=|\operatorname{Re}(e)|+|\operatorname{Im}(e)|$, has been introduced in [8]:

$$
\begin{aligned}
& \operatorname{STFT}_{M}(n, k) \\
& =\operatorname{median}\left\{\operatorname{Re}\left(x(n+m) e^{-j 2 \pi m k / N_{s}}\right), m \in\left[-\frac{N_{s}}{2}, \frac{N_{s}}{2}\right)\right\} \\
& \quad+j \cdot \operatorname{median}\left\{\operatorname{Im}\left(x(n+m) e^{-j 2 \pi m k / N_{s}}\right), m \in\left[-\frac{N_{s}}{2}, \frac{N_{s}}{2}\right)\right\} .
\end{aligned}
$$

If the signal is corrupted by a mixture of Gaussian and impulse noise, the L-estimate robust STFT is used. It is defined as [9]

$$
\begin{array}{r}
\operatorname{STFT}_{L}(n, k)=\sum_{i=-N_{s} / 2}^{N_{s} / 2-1} a_{i}\left(r_{i}(n, k)+j \cdot i_{i}(n, k)\right), \\
r_{i}(n, k) \in R(n, k), \\
R(n, k)=\left\{\operatorname{Re}\left(x(n+m) e^{-j 2 \pi m k / N_{s}}\right), m \in\left[-\frac{N_{s}}{2}, \frac{N_{s}}{2}\right)\right\} \\
i_{i}(n, k) \in I(n, k), \\
I(n, k)=\left\{\operatorname{Im}\left(x(n+m) e^{-j 2 \pi m k / N_{s}}\right), m \in\left[-\frac{N_{s}}{2}, \frac{N_{s}}{2}\right)\right\},
\end{array}
$$

where the elements $r_{i}(n, k)$ and $i_{i}(n, k)$ are sorted in nondecreasing order as $r_{i}(n, k) \leq r_{i+1}(n, k)$ and $i_{i}(n, k) \leq i_{i+1}(n, k)$, respectively. The coefficients $a_{i}$ are given as

$a_{i}= \begin{cases}\frac{1}{N_{s}(1-2 \alpha)+4 \alpha}, & \text { for } i \in\left[\left(N_{s}-2\right) \alpha, \alpha\left(2-N_{s}\right)+N_{s}-1\right], \\ 0, & \text { elsewhere, }\end{cases}$

where $N_{s}$ is even, while the parameter $\alpha$ takes values within the range $[0,1 / 2]$. For $\alpha=0$ and $\alpha=1 / 2$ the standard STFT and the marginal median STFT are obtained, respectively. Higher value of $\alpha$ provides an enhanced reduction of heavytailed noise, while smaller value of $\alpha$ improves spectral characteristics. Thus, depending on the application, the value of parameter $\alpha$ should be chosen to provide good trade-off between these requirements.

The robust spectrogram is obtained as

$$
\operatorname{SPEC}_{h}(n, k)=\operatorname{Re}\left\{\operatorname{STFT}_{h}(n, k)\right\}^{2}+\operatorname{Im}\left\{\operatorname{STFT}_{h}(n, k)\right\}^{2},
$$

where $\mathrm{STFT}_{h}$ may be $\mathrm{STFT}_{M}$ or $\mathrm{STFT}_{L}$.

2.2. Robust Quadratic Time-Frequency Distributions. The previous concept has been extended to the Wigner distribution. The WD can be obtained as a solution of the following optimization problem:

$$
\begin{gathered}
\mathrm{WD}(n, k)=\arg \min _{\mu} \sum_{m=-N_{s} / 2}^{N_{s} / 2-1} F(e(n, k, m)), \\
e(n, k, m)=\operatorname{Re}\left\{x(n+m) \bar{x}(n-m) e^{-j 4 \pi m k / N_{s}}-\mu\right\},
\end{gathered}
$$

where $\bar{x}$ denotes the complex conjugate of $x$. For the loss function $F(e)=|e|^{2}$, the standard WD follows:

$$
\begin{array}{r}
\mathrm{WD}_{S}(n, k)=\operatorname{mean}\left\{\operatorname{Re}\left\{x(n+m) \bar{x}(n-m) e^{-j 4 \pi m k / N_{s}}\right\}\right\}, \\
m \in\left[-\frac{N_{s}}{2}, \frac{N_{s}}{2}\right) .
\end{array}
$$


The median-based robust WD has been defined in [8] as a solution of optimization problem in (8), for the loss function $F(e)=|e|$ :

$$
\begin{array}{r}
\mathrm{WD}_{M}(n, k)=\operatorname{median}\left\{\operatorname{Re}\left\{x(n+m) \bar{x}(n-m) e^{-j 4 \pi k m / N_{s}}\right\}\right\}, \\
m \in\left[-\frac{N_{s}}{2}, \frac{N_{s}}{2}\right) .
\end{array}
$$

The L-estimate robust WD can be written as [9]

$$
\begin{gathered}
\mathrm{WD}_{L}(n, k)=\sum_{i=-N_{s} / 2}^{N_{s} / 2-1} a_{i} r_{i}(n, k), \\
r_{i}(n, k) \in R(n, k)=\left\{\operatorname{Re}\left(x(n+m) \bar{x}(n-m) e^{-j 4 \pi m k / N_{s}}\right)\right\}, \\
m \in\left[-\frac{N_{s}}{2}, \frac{N_{s}}{2}\right),
\end{gathered}
$$

where the elements $r_{i}(n, k)$ are sorted in nondecreasing order as $r_{i}(n, k) \leq r_{i+1}(n, k)$, while the coefficients $a_{i}$ are defined by (6). The marginal median WD follows for $\alpha=1 / 2$.

The WD can be calculated by using STFT:

$$
\operatorname{RWD}_{h}(n, k)=\sum_{l=-N_{s} / 2}^{N s / 2-1} \operatorname{STFT}_{h}(n, k+l) \overline{\operatorname{STFT}}_{h}(n, k-l),
$$

where $\mathrm{STFT}_{h}$ can be the median-based STFT or the Lestimate STFT.

The robust S-method has been introduced in order to reduce or remove cross-terms in the robust WD. It can be written in the following form [12]:

$$
\operatorname{RSM}_{h}(n, k)=\sum_{l=-L}^{L} P(l) \operatorname{STFT}_{h}(n, k+l) \overline{\operatorname{STFT}}_{h}(n, k-l),
$$

where $P(l)$ is a frequency domain window with its width equal to $2 L+1$. For $L=0$ and $L=N_{s} / 2$ the robust spectrogram and the robust WD are obtained, respectively. More details about parameter $L$ selection can be found in [5].

\section{Robust Complex-Lag Time-Frequency Distributions}

The time-frequency distributions with complex-lag argument have been introduced for signals with fast varying instantaneous frequency. The general form of the standard $N$ th-order complex-lag time-frequency distribution can be written as [18]

$$
\begin{aligned}
\operatorname{CTD}^{N}(n, k) & =\sum_{m=-N_{s} / 2}^{N_{s} / 2-1} x(n+m) \bar{x}(n-m) c(n, m) e^{-j\left(2 \pi / N_{s}\right) N m k} \\
& =\sum_{l=-N_{s} / 2}^{N_{s} / 2-1} \operatorname{WD}(n, k+l) \operatorname{CF}(n, k-l),
\end{aligned}
$$

TABLE 1: Spread factors for some time-frequency distributions.

\begin{tabular}{ll}
\hline Distribution & Spread factor \\
\hline $\begin{array}{l}\mathrm{CTD}^{2}, \text { that is, }(N=2) \\
\mathrm{WD}\end{array}$ & $Q(t, \tau)=\phi^{(3)}(t) \frac{\tau^{3}}{2^{2} 3 !}+\phi^{(5)}(t) \frac{\tau^{5}}{2^{4} 5 !}+\cdots$ \\
\hline $\mathrm{CTD}^{4}(N=4)$ & $Q(t, \tau)=\phi^{(5)}(t) \frac{\tau^{5}}{4^{4} 5 !}+\phi^{(9)}(t) \frac{\tau^{9}}{4^{8} 9 !}+\cdots$ \\
\hline $\mathrm{CTD}^{6}(N=6)$ & $Q(t, \tau)=\phi^{(7)}(t) \frac{\tau^{7}}{6^{6} 7 !}+\phi^{(13)}(t) \frac{\tau^{13}}{6^{12} 13 !}+\cdots$ \\
\hline $\mathrm{CTD}^{N}$ & $Q(t, \tau)=$ \\
\hline$\phi^{N+1}(t) \frac{\tau^{N+1}}{N^{N}(N+1) !}+\phi^{2 N+1}(t) \frac{\tau^{2 N+1}}{N^{2 N}(2 N+1) !}+\cdots$
\end{tabular}

where the standard WD is given by $(9)$, while $C F(n, k)$ is the Fourier transform of the complex-lag signal moment:

$$
c(n, m)=\prod_{p=1}^{N / 2-1} x^{\bar{w}_{N, p}}\left(n+w_{N, p} m\right) x^{-\bar{w}_{N, p}}\left(n-w_{N, p} m\right),
$$

where $N$ is an even number, representing the distribution order, while the quantity $w_{N, p}=e^{j 2 \pi p / N}, p=1, \ldots, N / 2-1$ defines the equidistant roots on the unit circle. It has been shown [17-19] that the distribution defined by (14) can provide an arbitrarily high concentration by increasing $N$. Namely, the complex-lag distributions significantly reduce the spread factor produced by higher phase derivatives. For example, the fourth-order distribution:

$$
\begin{aligned}
& \operatorname{CTD}^{4}(n, k) \\
& =\sum_{m=-N_{s} / 2}^{N_{s} / 2-1} x(n+m) \bar{x}(n-m) x^{-j}(n+j m) \\
& \quad \times x^{j}(n-j m) e^{-j\left(2 \pi / N_{s}\right) 4 m k}
\end{aligned}
$$

contains the number of spread terms that is twice smaller than for the WD (Table 1). Furthermore, the sixth-order distribution is obtained for the roots $\left\{w_{1}, w_{2}\right\}=\{1 / 2+$ $j \sqrt{3} / 2,-1 / 2+j \sqrt{3} / 2\}$ :

$$
\begin{aligned}
& \operatorname{CTD}^{6}(n, k) \\
& =\sum_{m=-N_{s} / 2}^{N_{s} / 2} s(n+m) s^{-1}(n-m) \times\left(s\left(n+w_{1} m\right) s^{-1}\left(n-w_{1} m\right)\right)^{w_{1}^{*}} \\
& \quad \times\left(s\left(n+w_{2} m\right) s^{-1}\left(n-w_{2} m\right)\right)^{w_{2}^{*}} e^{-j\left(2 \pi / N_{s}\right) 6 m k},
\end{aligned}
$$

and its spread factor is also given in Table 1. Note that, in general, the first term in the spread factor is of order $(N+1)$. 
In order to obtain $c(n, m)$, the signal with complex-lag argument $x\left(n \pm w_{N, p} m\right)$ is calculated by using the concept of analytic extension as follows:

$$
\begin{aligned}
x(n \pm & \left.w_{N, p} m\right) \\
& =\sum_{k=-N_{s} / 2}^{N_{s} / 2-1} X_{S}(k) e^{j\left(2 \pi / N_{s}\right)\left(n \pm w_{N, p} m\right) k} \\
& =\sum_{k=-N_{s} / 2}^{N_{s} / 2-1} X_{S}(k) e^{\mp\left(2 \pi / N_{s}\right) w i_{N, p} m k} e^{j\left(2 \pi / N_{s}\right)\left(n \pm w r_{N, p} m\right) k},
\end{aligned}
$$

where $w r_{N, p}=\operatorname{Re}\left\{w_{N, p}\right\}, w i_{N, p}=\operatorname{Im}\left\{w_{N, p}\right\}$, while $X_{S}(k)$ is the standard Fourier transform. The coordinate $m$ is multiplied by $w r_{N, p}$. The influence of this term can be such that an additional oversampling (or interpolations) of signal $x(n)$ is required.

By analogy with the standard CTD, the robust CTD can be defined as

$$
\operatorname{RCTD}^{N}(n, k)=\sum_{l=-N_{s} / 2}^{N_{s} / 2-1} \operatorname{RWD}(n, k+l) \operatorname{RCF}(n, k-l),
$$

where RWD is the robust WD, while robust CF (RCF) is obtained as a solution of the optimization problem:

$$
\begin{gathered}
\mathrm{CF}(n, k)=\arg \min _{\mu} \sum_{m=-N_{s} / 2}^{N_{s} / 2-1} F(e(n, k, m)), \\
e(n, k, m)=c(n, m) e^{-j\left(2 \pi / N_{s}\right) N m k}-\mu,
\end{gathered}
$$

for the loss function $F(e)=|e|$. The robust WD calculation has been already presented, while the robust CF can be obtained as a solution of nonlinear equation:

$$
\begin{aligned}
& \operatorname{RCF}(n, k) \\
& =\frac{1}{\sum_{m=-N_{s} / 2}^{N_{s} / 2-1}(1 /|e(n, k, m)|)} \\
& \quad \times \sum_{m=-N_{s} / 2}^{N_{s} / 2-1} \frac{1}{|e(n, k, m)|} c(n, m) e^{-j\left(2 \pi / N_{s}\right) N m k}, \\
& e(n, k, m)=c(n, m) e^{-j\left(2 \pi / N_{s}\right) N m k}-\operatorname{RCF}(n, k) .
\end{aligned}
$$

The iterative procedure for the nonlinear equation (21) is even more demanding than in the cases of robust STFT and robust WD calculations $[6,11]$. Namely, to calculate $c(n, m)$, the robust form of (18) has to be used. It requires an additional iterative procedure. Hence, the CF calculation requires nested iterative procedures, inappropriate for practical realization.

3.1. L-Estimate Form of the Robust CTD. The marginal median and the L-estimate approach can be used to overcome disadvantages of iterative procedure for the robust CTD calculation. In the sequel, only the L-estimate approach is considered, since the marginal median follows as a special case of the L-estimate forms for $\alpha=1 / 2$. Also, the L-estimates exhibit enhanced performance in the presence of mixture of Gaussian and impulse noise, common to real applications. Thus, the L-estimate approach is used to define the robust CTD.

Having in mind (19), the L-estimate robust CTD can be obtained as a convolution of the L-estimate robust WD and the L-estimate robust CF. By analogy with the robust WD, the L-estimate approach is used for the robust CF calculation, as follows:

$$
\operatorname{RCF}_{L}(n, k)=\sum_{i=-N_{s} / 2}^{N_{s} / 2-1} a_{i}\left(c r_{i}(n, m)+j \cdot c i_{i}(n, m)\right),
$$

where $c r_{i}(n, k)$ and $c i_{i}(n, k)$ are the elements of

$$
\begin{aligned}
& R(n, k)=\operatorname{Re}\left\{r c(n, m) e^{-j\left(2 \pi / N_{s}\right) N m k}, m \in\left[-\frac{N_{s}}{2}, \frac{N_{s}}{2}\right)\right\}, \\
& I(n, k)=\operatorname{Im}\left\{r c(n, m) e^{-j\left(2 \pi / N_{s}\right) N m k}, m \in\left[-\frac{N_{s}}{2}, \frac{N_{s}}{2}\right)\right\},
\end{aligned}
$$

respectively. They are sorted in nondecreasing order: $c r_{i}(n, k) \leq c r_{i+1}(n, k)$ and $c i_{i}(n, k) \leq c i_{i+1}(n, k)$. The coefficients $a_{i}$ are given by (6), while $r c(n, m)$ represent the robust complex-lag signal moment:

$$
\begin{gathered}
r c(n, m)=\prod_{p=1}^{N / 2-1} c r_{p}(n, m) c i_{p}(n, m), \\
c r_{p}(n, m)=e^{j w r_{N, p} \operatorname{angle}\left(x\left(n+w_{N, p} m\right) / x\left(n-w_{N, p} m\right)\right)}, \\
c i_{p}(n, m)=e^{j w i_{N, p} \log \left|x\left(n-w_{N, p} m\right) / x\left(n+w_{N, p} m\right)\right|} .
\end{gathered}
$$

The numerical realization is simplified by using the angle and $\log |\cdot|$ functions. Namely, for a signal in the form $x(t)=$ $A e^{j \phi(t)}$, the amplitude modulation terms that may appear in the calculation of $c r_{p}(n, m)$ and $c i_{p}(n, m)$ are eliminated, [18]. Also, calculation of signal raised to power $j$ is avoided by using the exponential with $\log |\cdot|$ function, [14]. The signal with the complex-lag argument is obtained as

$$
x_{L}\left(n \pm w_{N, p} m\right)=\sum_{k=-N_{s} / 2}^{N_{s} / 2-1} X_{L}(k) e^{j 2 \pi\left(n \pm w_{N, p} m\right) k},
$$

where $X_{L}(k)$ represents the L-estimate of Fourier transform:

$$
X_{L}(k)=\sum_{i=-N_{s} / 2}^{N_{s} / 2-1} a_{i}\left(r_{i}(k)+j \cdot i_{i}(k)\right)
$$

where $r_{i}(k) \in R(k), R(k)=\left\{\operatorname{Re}\left(x(n) e^{-j 2 \pi k n / N_{s}}\right), n \in\left[0, N_{s}-\right.\right.$ $1)\}$ and $i_{i}(k) \in I(k), I(k)=\left\{\operatorname{Im}\left(x(n) e^{-j 2 \pi n k / N_{s}}\right), n \in\right.$ $\left.\left[0, N_{s}-1\right)\right\}$ are such that $r_{i}(k) \leq r_{i+1}(k)$ and $i_{i}(k) \leq i_{i+1}(k)$.

Finally, the L-estimate of complex-lag time-frequency distribution is:

$$
\operatorname{RCTD}_{L}^{N}(n, k)=\mathrm{WD}_{L}(n, k)_{* k} \operatorname{RCF}_{L}(n, k),
$$

where $\mathrm{WD}_{L}$ represents the L-estimate robust $\mathrm{WD}$. 


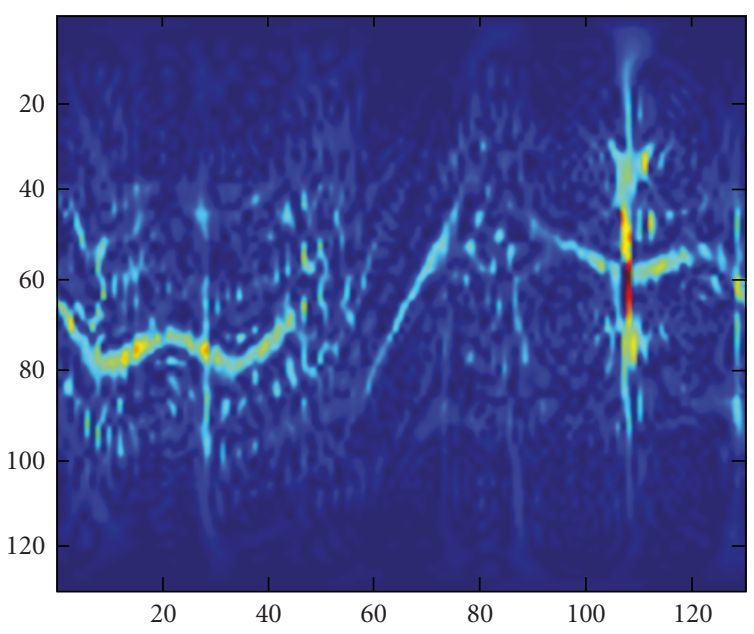

(a)

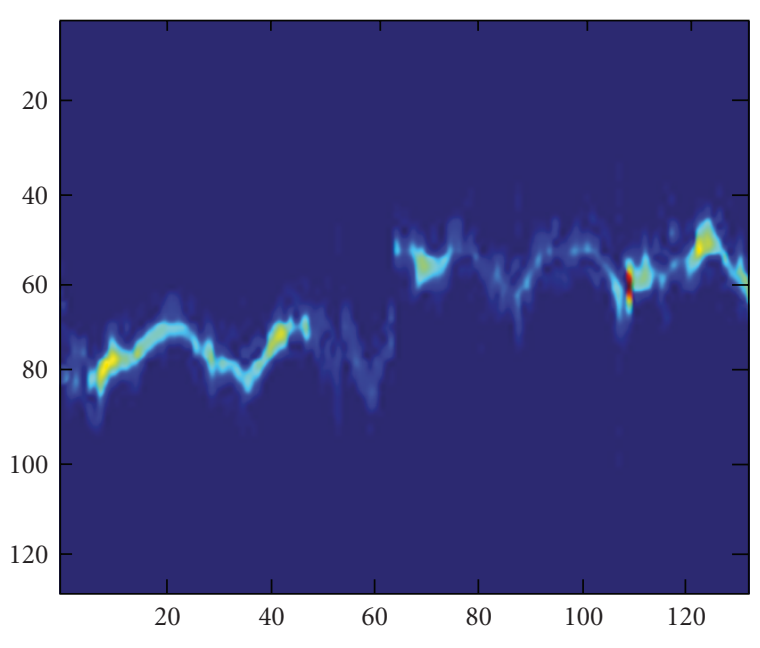

(c)

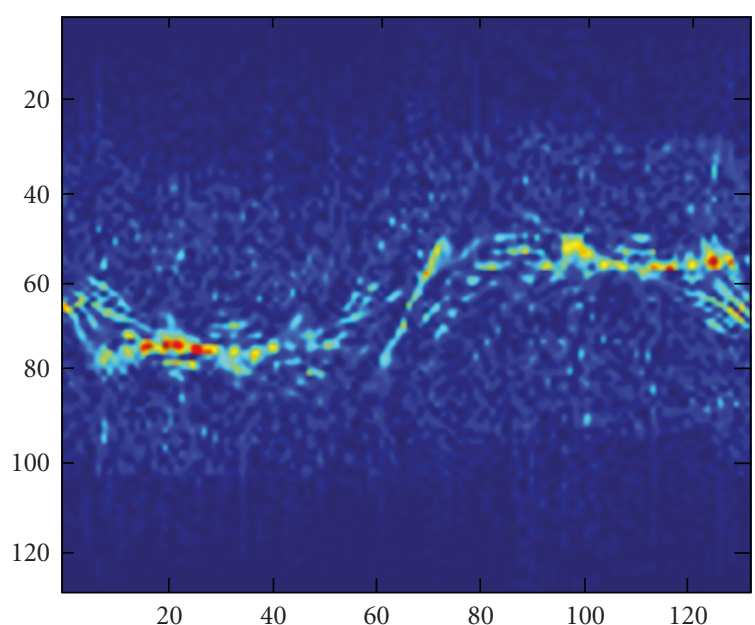

(b)

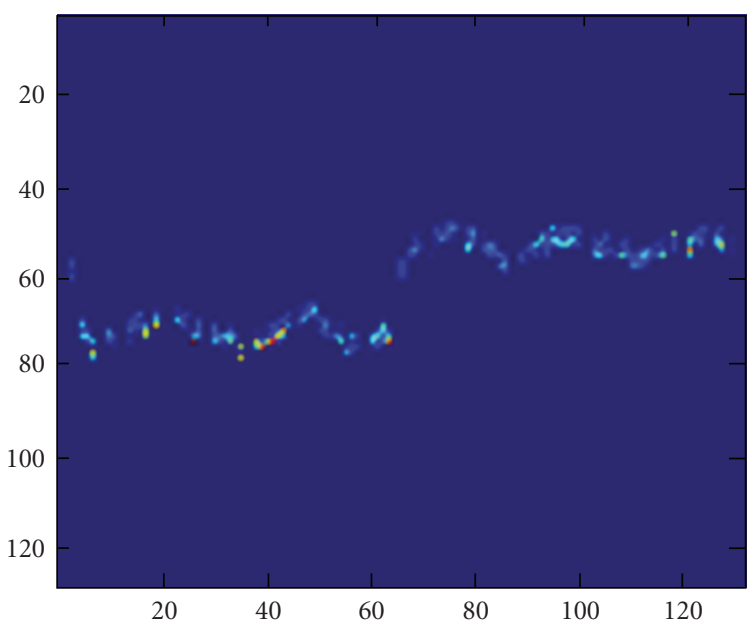

(d)

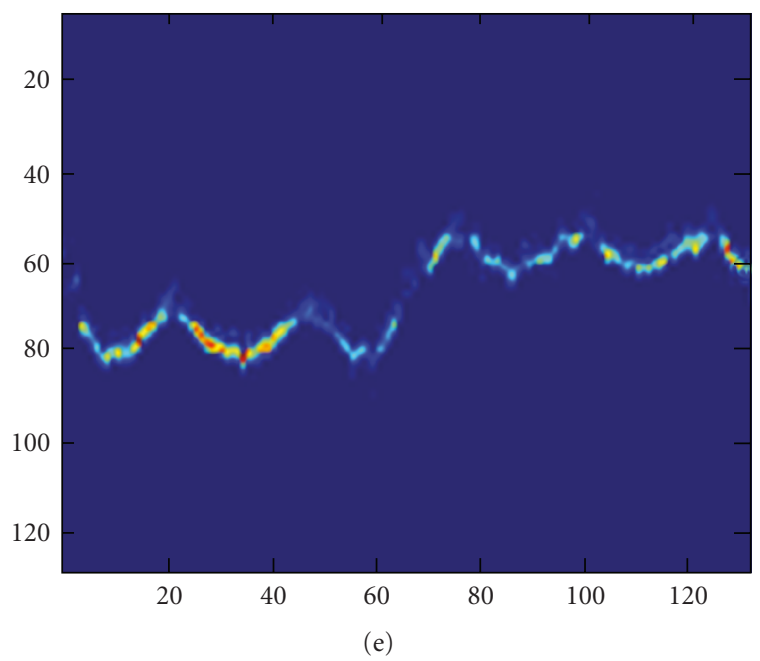

FIGURE 1: Time-frequency representations for signal $x_{1}(n)$ by using (a) the standard WD, (b) the L-estimate WD, (c) the standard CTD ${ }^{N=4}$, (d) the marginal median $\operatorname{RCTD}^{N=4}$, and (e) the L-estimate $\operatorname{RCTD}^{N=4}$. 
3.2. Robust CTD Form for Multicomponent Signals. Note that the robust CTD form (defined by (27)) can be used for monocomponent signals. However, in the case of multicomponent signal: $x(n)=\sum_{q=1}^{Q} s_{q}(n)+v(n)$, the cross-terms appear. Thus, it is necessary to modify (27). The robust S-method, as a cross-terms free distribution, will be used instead of the robust WD, while a modification providing a cross-terms free robust $\mathrm{CF}$ should be introduced. In that sense, the signal with complex-lag argument is separately calculated for each component. The component separation is performed by using the robust STFT. Namely, the qth signal component is obtained as

$$
\begin{aligned}
x_{L}(n & \left. \pm w_{N, p} m\right)_{q} \\
& =\sum_{k=-W_{q}}^{W_{q}} \operatorname{STFT}_{L}\left(n, k+k_{q}(n)\right) e^{j\left(k+k_{q}(n)\right)\left(n \pm w_{N, p} m\right)},
\end{aligned}
$$

where $k_{q}(n)=\arg \left\{\max _{k} \operatorname{STFT}_{L}(n, k)\right\}$ is the position of the $q$ th signal component maximum in the L-estimate robust STFT. It is assumed that the $q$-th signal component is of $2 W_{q}+1$ width; that is, it is within the region $\left[k_{q}(n)-W_{q}\right.$, $\left.k_{q}(n)+W_{q}\right]$. Observe that the cross-terms will be avoided, if the distance between signal components is higher than $2 W_{q}$ (see [14] for details). After the first signal component is obtained, the values of $\operatorname{STFT}_{L}(n, k)$ within the region $\left[k_{q}(n)\right.$ $\left.W_{q}, k_{q}(n)+W_{q}\right]$ will be set to 0 . Then, this procedure is repeated for other components.

Furthermore, for the $q$ th signal component the complexlag signal moments $\operatorname{cr}(n, m)_{q}$ and $c i(n, m)_{q}$ are

$$
\begin{aligned}
& \operatorname{cr}(n, m)_{q} \\
& =\prod_{p=1}^{N / 2-1} c r_{p}(n, m)_{q}=\prod_{p=1}^{N / 2-1} e^{j w r_{N, p} \operatorname{angle}\left(x_{L}\left(n+w_{N, p} m\right)_{q} / x_{L}\left(n-w_{N, p} m\right)_{q}\right)}, \\
& \operatorname{ci}(n, m)_{q} \\
& =\prod_{p=1}^{N / 2-1} c i_{p}(n, m)_{q}=\prod_{p=1}^{N / 2-1} e^{j w i_{N, p} \log \left|x_{L}\left(n-w_{N, p} m\right)_{q} / x_{L}\left(n+w_{N, p} m\right)_{q}\right|} .
\end{aligned}
$$

For all signal components we have $\operatorname{cr}(n, m)=\sum_{q=1}^{Q} \operatorname{cr}(n, m)_{q}$ and $c i(n, m)=\sum_{q=1}^{Q} c i(n, m)_{q}$. By using the L-estimate approach, two corresponding robust CFs can be defined as

$$
\begin{aligned}
& \operatorname{RCF}_{L}(n, k)=\sum_{i=-N_{s} / 2}^{N_{s} / 2-1} a_{i}\left(v r_{i}(n, m)+j v i_{i}(n, m)\right), \\
& \operatorname{RCF}_{L}(n, k)=\sum_{i=-N_{s} / 2}^{N_{s} / 2-1} a_{i}\left(u r_{i}(n, m)+j u i_{i}(n, m)\right),
\end{aligned}
$$

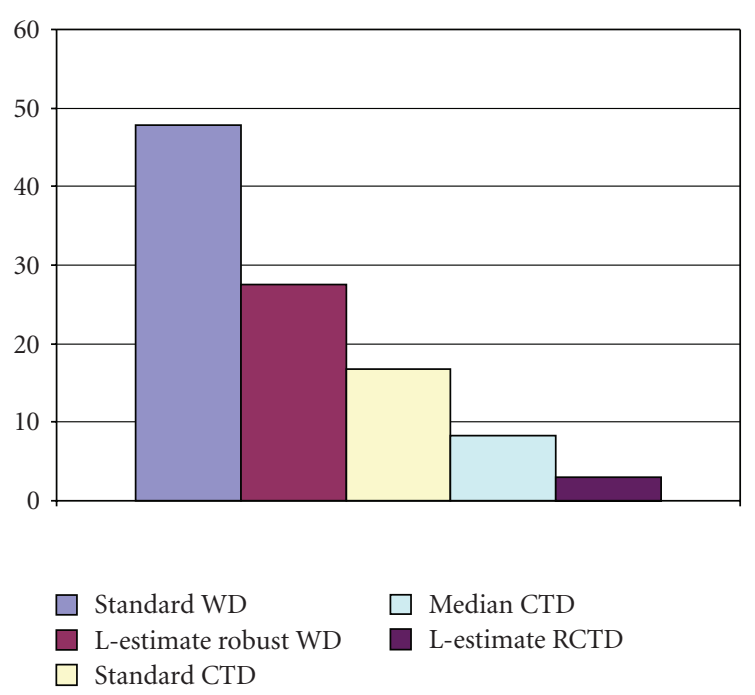

FIGURE 2: MSE of instantaneous frequency estimation in the presence of heavy-tailed noise.

where $v r_{i}(n, k)$ and $v i_{i}(n, k)$ (sorted in nondescending order) are elements of:

$$
\begin{gathered}
R v(n, k)=\operatorname{Re}\left\{c r(n, m) e^{-j\left(2 \pi / N_{s}\right) N m k}, m \in\left[-\frac{N_{s}}{2}, \frac{N_{s}}{2}\right)\right\}, \\
I v(n, k)=\operatorname{Im}\left\{c r(n, m) e^{-j\left(2 \pi / N_{s}\right) N m k}, m \in\left[-\frac{N_{s}}{2}, \frac{N_{s}}{2}\right)\right\},
\end{gathered}
$$

respectively. Similarly, $u r_{i}(n, k)$ and $u i_{i}(n, k)$ are elements of:

$$
\begin{aligned}
& R u(n, k)=\operatorname{Re}\left\{c i(n, m) e^{-j\left(2 \pi / N_{s}\right) N m k}, m \in\left[-\frac{N_{s}}{2}, \frac{N_{s}}{2}\right)\right\}, \\
& I u(n, k)=\operatorname{Im}\left\{c i(n, m) e^{-j\left(2 \pi / N_{s}\right) N m k}, m \in\left[-\frac{N_{s}}{2}, \frac{N_{s}}{2}\right)\right\},
\end{aligned}
$$

where $u r_{i}(n, k) \leq u r_{i+1}(n, k)$ and $u i_{i}(n, k) \leq u i_{i+1}(n, k)$.

The cross-terms free robust CF is:

$$
\widetilde{\operatorname{RCF}}_{L}(n, k)=\sum_{l=-L}^{L} P(l) \mathrm{CF} r_{L}(n, k+l) \mathrm{CF} i_{L}(n, k-l) .
$$

Finally, the L-estimate robust CTD for multicomponent signals can be written in the form:

$$
\operatorname{RCTD}_{L}^{N}(n, k)=\sum_{l=-L}^{L} P(l) \operatorname{SM}_{L}(n, k+l) \widetilde{\operatorname{RCF}}_{L}(n, k-l)
$$

The role of window $P(l)$ is same as in the S-method. 


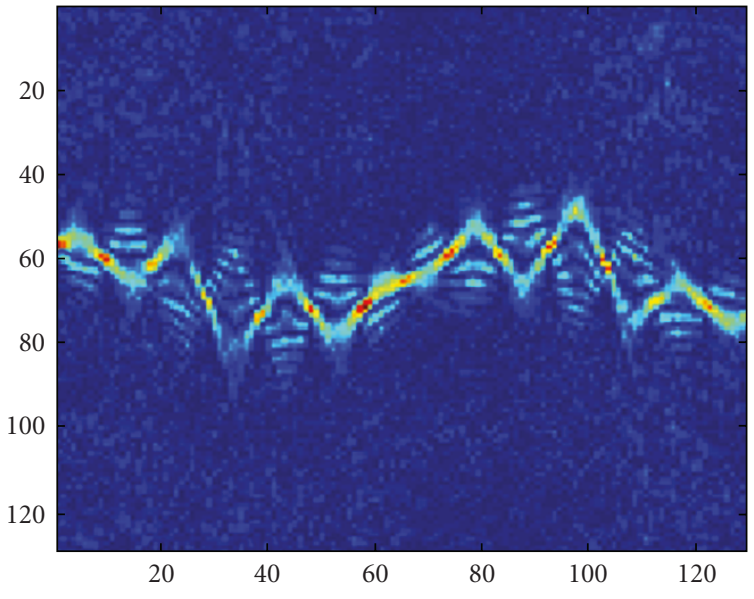

(a)

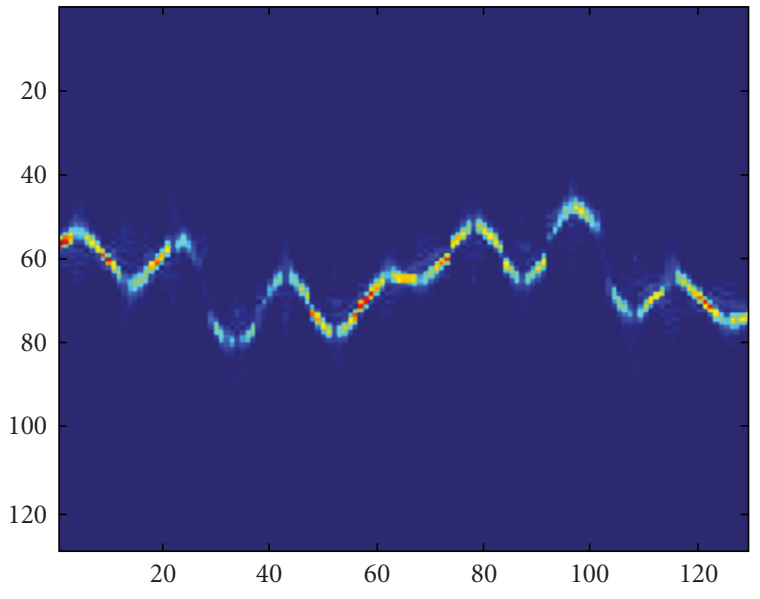

(b)

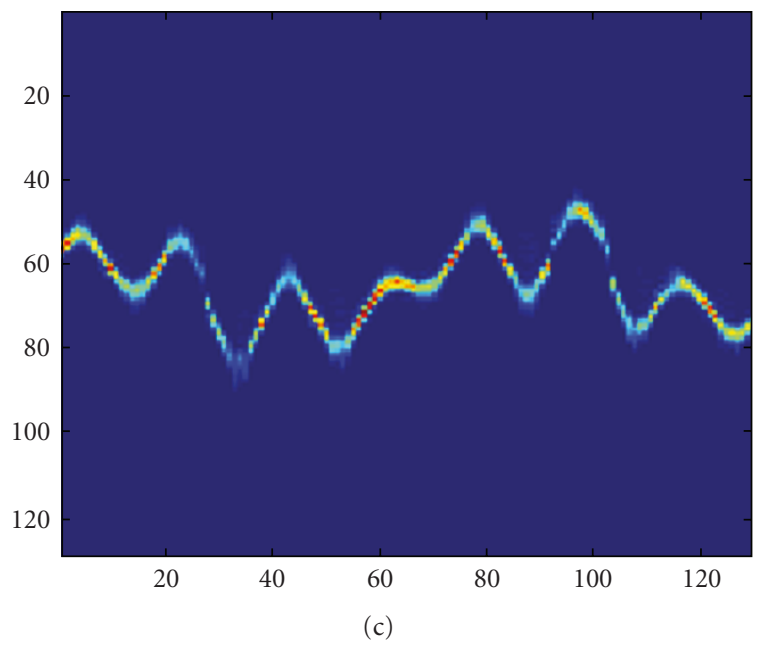

FIgURE 3: Time-frequency representations for signal $y(n)$ by using: (a) the L-estimate WD, (b) the L-estimate RCTD $^{N=4}$, and (c) the Lestimate $\operatorname{RCTD}^{N=6}$.

\section{Examples}

Highly nonstationary signals with fast varying instantaneous frequencies are considered. The signals are corrupted with heavy-tailed noise. The standard and the robust forms of CTD are considered and compared with corresponding forms of the Wigner distribution.

Example 1. Consider a noisy signal:

$$
x(n)=e^{2 j(6 \cos (\pi n)+2 / 3 \cos (3 \pi n)+2 / 3 \cos (5 \pi n))}+\xi(n),
$$

where $\xi(n)$ is heavy-tailed complex valued noise (cube of Gaussian noise):

$$
\xi(n)=0.5 \xi_{1}^{3}(n)+0.5 j \xi_{2}^{3}(n),
$$

where $\xi_{1}(n)$ and $\xi_{2}(n)$ are mutually independent Gaussian noises (zero mean with variance equal to 1 ). The time interval $t \in[-2,2]$, with sampling rate $T=1 / 128$, is used. The Gaussian window of $N_{s}=128$ width is applied in all cases.
The L-estimate forms are calculated by using parameter $\alpha=3 / 8$ for all distributions. Namely, this value provides satisfying trade-off between noise reduction and distribution concentration. For a given signal, the standard WD, the L-estimate WD, the standard $\mathrm{CTD}^{N=4}$, the marginal median RCTD $^{N=4}$ (obtained according to (27) with $\alpha=1 / 2$ ), and the L-estimate $\operatorname{RCTD}^{N=4}$ are shown in Figure 1. Since the signal $x_{1}(n)$ has a fast varying IF, both the standard WD (Figure 1(a)) and the L-estimate WD (Figure 1(b)) yield poor results. The fourth-order CTD is introduced to improve concentration. However, as expected, the standard $\mathrm{CTD}^{N=4}$ (Figure $1(\mathrm{c})$ ) contains significant disturbances, due to the presence of strong heavy-tailed noise. Furthermore, the robust complex-lag distribution forms are considered. Thus, the results are improved by using the median $\operatorname{RCTD}^{N=4}$ (Figure $1(\mathrm{~d})$ ), but the accuracy of estimation is still not satisfactory. The best result in this example, regarding representation and IF estimation precision, is achieved by using the L-estimate $\operatorname{RCTD}^{N=4}$ (Figure 1(e)). 


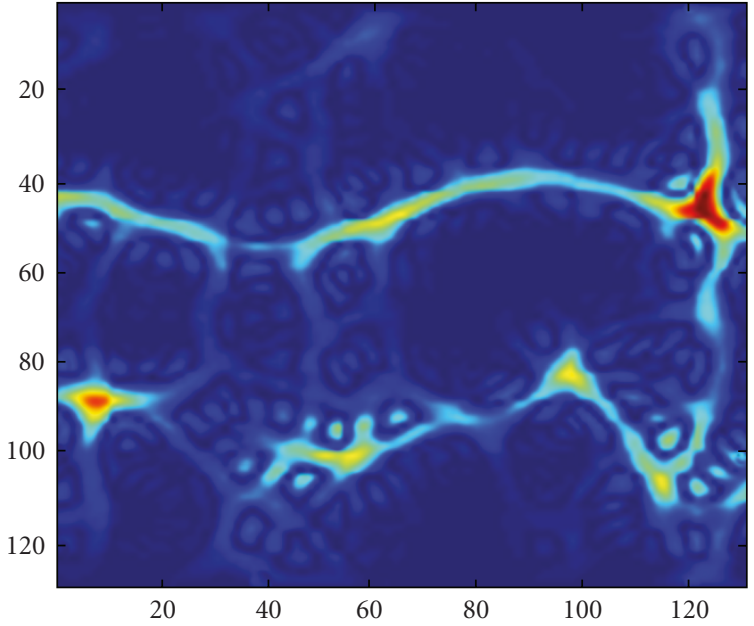

(a)

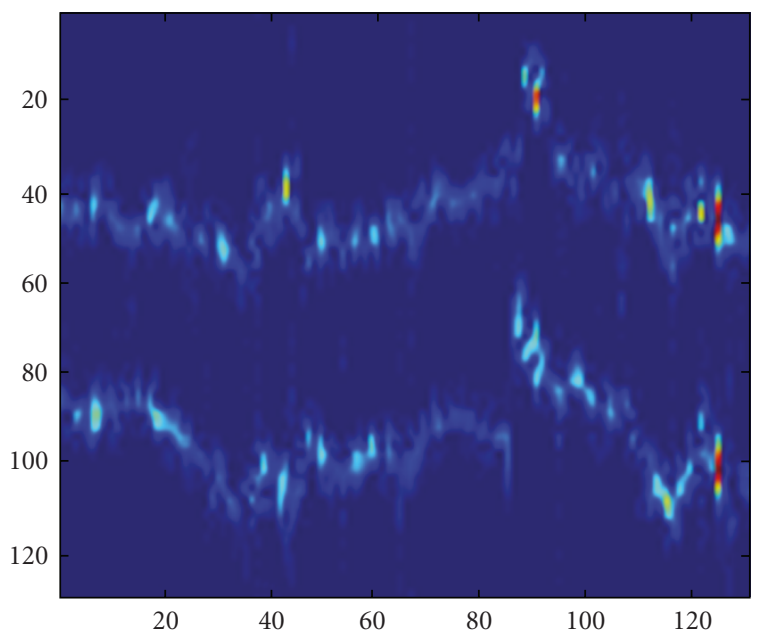

(c)

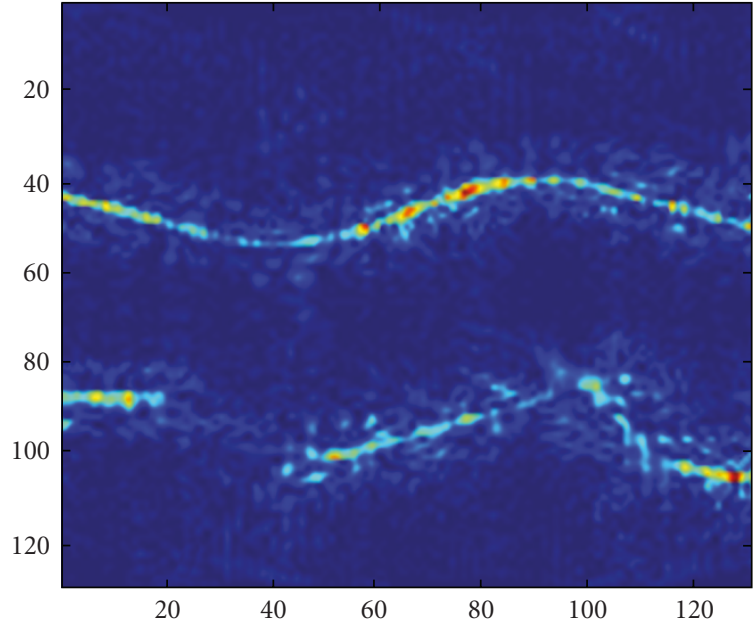

(b)

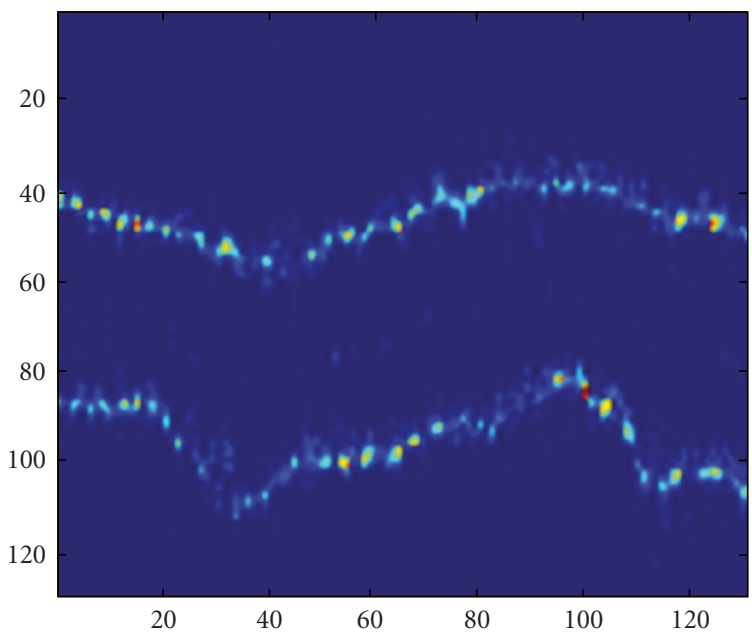

(d)

Figure 4: Time-frequency representation for multicomponent signal $x(n)$ by using (a) the standard SM, (b) the L-estimate SM, (c) the standard $\mathrm{CTD}^{N=4}$, and (d) the L-estimate $\mathrm{RCTD}^{N=4}$.

The mean squared error (MSE) is used as a quantitative measure of performance, for all distributions:

$$
\operatorname{MSE}=\frac{1}{N_{s}} \sum_{n=1}^{N_{s}}|\varphi(n)-\hat{\varphi}(n)|^{2}
$$

where $\varphi(n)$ is the true IF, while $\hat{\varphi}(n)$ is the estimated IF: $\hat{\varphi}(n)=\arg \left\{\max _{k} \operatorname{STFT}(n, k)\right\}$. The mean values of MSEs are given in Figure 2 for 100 realizationsof noises. Note that the L-estimate RCTD ${ }^{N=4}$ provides the lowest MSE.

In the presence of Gaussian noise, the performance of L-estimate $\mathrm{RCTD}^{N=4}$ is similar to the performance of the standard $\mathrm{CTD}^{N=4}$. The MSEs of IF estimation, calculated as a mean value for 100 realizations of Gaussian noises, are given in Table 2.

Example 2. This example aims to illustrate how the distribution order has to be increased to achieve concentration
TABle 2: The MSE of instantaneous frequency estimation in the presence of Gaussian noise.

\begin{tabular}{lc}
\hline Distribution & MSE \\
\hline Standard CTD & 2.45 \\
L-estimate RCTD $^{N=4}$ & 2.56 \\
\hline
\end{tabular}

improvement. Namely, in the case of signal with IF variations that are faster than in the previous example, for example,

$$
y(n)=e^{2 j(3 \cos (1.5 \pi n)+2 / 3 \cos (7 \pi n)+1 / 2 \cos (5 \pi n))}+\xi(n),
$$

the fourth-order distribution is no longer optimal. Thus, the concentration can be improved by using the sixthorder distribution. The noise $\xi(n)$ is the same as in the previous example. The L-estimate RWD, the L-estimate $\operatorname{RCTD}^{N=4}$, and L-estimate $\mathrm{RCTD}^{N=6}$ are shown in Figure 3. The parameter $\alpha=3 / 8$ is used in all cases. 
TABLE 3: The MSE of instantaneous frequency estimation.

\begin{tabular}{lc}
\hline Distribution & MSE \\
\hline L-estimate WD & 51.83 \\
L-estimate RCTD & \\
L=4 & 8.52 \\
L-estimate RCTD $^{N=6}$ & 3.45 \\
\hline
\end{tabular}

As expected, the L-estimate WD is not suitable for analysis. Significant improvements were obtained with the L-estimate $\mathrm{RCTD}^{N=4}$, while the best results are achieved by using the L-estimate $\mathrm{RCTD}^{N=6}$ in this example. The MSE of instantaneous frequency estimation is given in Table 3 for 100 realizations of noises. Note that the lowest MSE is obtained for the L-estimate RCTD ${ }^{N=6}$ distribution.

Example 3. Consider a noisy multicomponent signal:

$$
\begin{aligned}
z(n)= & e^{j(6 \cos ((3 / 2) \pi n)-(4 / 3) \cos (7 \pi n)+\cos (5 \pi n)+15 \pi n)} \\
& +e^{j\left(7,5 \pi\left(0.5 n^{4}-0.8 \pi n^{2}-8,5 \pi n\right)\right.} \\
& +0.5 \xi_{1}^{3}(n)+0.5 j \xi_{2}^{3}(n)
\end{aligned}
$$

where $\xi_{1}(n)$ and $\xi_{2}(n)$ represent Gaussian noises. The same parameters for time interval, window, and noise strength are used as in the Example 2. The results for the standard SM, the L-estimate $\mathrm{SM}$, the standard $\mathrm{CTD}^{N=4}$, and the L-estimate RCTD $^{N=4}$ are shown in Figure 4.

Note that both the standard SM and the standard $\mathrm{CTD}^{N=4}$ cannot provide satisfactory results due to the presence of impulse noise. The L-estimate SM provides good estimation for the component with slow varying instantaneous frequency (Figure 4(b)). However, it cannot follow the fast IF variationsfor the second signal component. The L-estimate RCTD $^{N=4}$ provides satisfying concentration for both components (Figure 4(d)).

\section{Conclusion}

The L-estimate-based robust Nth-order complex-lag timefrequency distribution has been proposed. It provides an efficient estimation for nonstationary signals corrupted with a mixture of Gaussian and heavy-tailed impulse noise. Additionally, we proposed the modified L-estimate robust CTD form that provides a cross-terms free representation for multicomponent signals.

The L-estimate and standard distribution approaches could be combined in some future work to reduce the calculation complexity. Also, the future research could be focused to generalize the proposed approach to the class of complex-time distributions based on the ambiguity domain [20].

\section{Acknowledgment}

This work is supported by the Ministry of Education and Science of Montenegro.

\section{References}

[1] L. Cohen, Time-Frequency Analysis, Prentice-Hall, Englewood Cliffs, NJ, USA, 1995.

[2] B. Boashash, Time-Frequency Analysis and Processing, Elsevier, Amsterdam, The Netherlands, 2003.

[3] B. Boashash, "Estimating and interpreting the instantaneous frequency of a signal-part 1: fundamentals," Proceedings of the IEEE, vol. 80, no. 4, pp. 520-538, 1992.

[4] F. Hlawatsch and G. F. Boudreaux-Bartels, "Linear and quadratic time-frequency signal representations," IEEE Signal Processing Magazine, vol. 9, no. 2, pp. 21-67, 1992.

[5] LJ. Stanković, "A method for time-frequency analysis," IEEE Transactions on Signal Processing, vol. 42, no. 1, pp. 225-229, 1994.

[6] V. Katkovnik, "Robust M-periodogram," IEEE Transactions on Signal Processing, vol. 46, no. 11, pp. 3104-3109, 1998.

[7] V. Katkovnik, I. Djurović, and LJ. Stanković, "Instantaneous frequency estimation using robust spectrogram with varying window length," AEU-International Journal of Electronics and Communications, vol. 54, no. 4, pp. 193-202, 2000.

[8] I. Djurovi, V. Katkovnik, and LJ. Stanković, "Median filter based realizations of the robust time-frequency distributions," Signal Processing, vol. 81, no. 8, pp. 1771-1776, 2001.

[9] I. Djurović, LJ. Stanković, and J. F. Böhme, "Robust Lestimation based forms of signal transforms and timefrequency representations," IEEE Transactions on Signal Processing, vol. 51, no. 7, pp. 1753-1761, 2003.

[10] V. Katkovnik, I. Djurović, and LJ. Stanković, "Robust timefrequency distributions," in Time-Frequency Signal Analysis and Applications, B. Boashash, Ed., pp. 392-399, Elsevier, Amsterdam, The Netherlands, 2003.

[11] I. Djurović and LJ. Stanković, "Robust wigner distribution with application to the instantaneous frequency estimation," IEEE Transactions on Signal Processing, vol. 49, no. 12, pp. 2985-2993, 2001.

[12] I. Djurović, LJ. Stanković, and B. Barkat, "Robust timefrequency distributions based on the robust short time Fourier transform," Annales des Telecommunications/Annals of Telecommunications, vol. 60, no. 5-6, pp. 681-697, 2005.

[13] S. Stanković and LJ. Stanković, "Introducing time-frequency distribution with a 'complex-time' argument," Electronics Letters, vol. 32, no. 14, pp. 1265-1267, 1996.

[14] LJ. Stanković, "Time-frequency distributions with complex argument," IEEE Transactions on Signal Processing, vol. 50, no. 3, pp. 475-486, 2002.

[15] M. Morelande, B. Senadji, and B. Boashash, "Complex-lag polynomial Wigner-Ville distribution," in Proceedings of the 10th IEEE Annual Conference on Speech and Image Technologies for Computing and Telecommunications (TENCON '97), vol. 1, pp. 43-46, Brisbane, Australia, December 1997.

[16] G. Viswanath and T. V. Sreenivas, "IF estimation using higher order TFRs," Signal Processing, vol. 82, no. 2, pp. 127-132, 2002.

[17] C. Cornu, S. Stanković, C. Ioana, A. Quinquis, and LJ. Stanković, "Generalized representation of phase derivatives for regular signals," IEEE Transactions on Signal Processing, vol. 55, no. 10, pp. 4831-4838, 2007.

[18] S. Stanković, N. Žarić, I. Orović, and C. Ioana, "General form of time-frequency distribution with complex-lag argument," Electronics Letters, vol. 44, no. 11, pp. 699-701, 2008. 
[19] S. Stanković and I. Orovic, "Effects of Cauchy integral formula discretization on the precision of IF estimation: unified approach to complex-lag distribution and its L-form," IEEE Signal Processing Letters, vol. 16, no. 4, pp. 327-330, 2009.

[20] I. Orović and S. Stanković, "A class of highly concentrated time-frequency distributions based on the ambiguity domain representation and complex-lag moment," EURASIP Journal on Advances in Signal Processing, vol. 2009, Article ID 935314, 9 pages, 2009.

[21] T. A. H. Li and K. A. I. S. Song, "Estimation of the parameters of sinusoidal signals in non-Gaussian noise," IEEE Transactions on Signal Processing, vol. 57, no. 1, pp. 62-72, 2009. 